\title{
Interconnectable Dynamic Compression Bioreactors for Combinatorial Screening of Cell Mechanobiology in Three Dimensions
}

\author{
Jungmok Seo, ${ }^{\dagger, \downarrow, \S, \$ \neq}$ Jung-Youn Shin, $\|,+\neq$ Jeroen Leijten, ${ }^{\dagger, \downarrow \perp}$ Oju Jeon, $\|$ Ayça Bal Öztürk, ${ }^{\dagger, \ddagger}$ Jeroen Rouwkema, ${ }^{\#}$

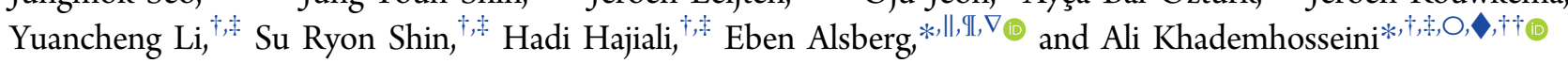 \\ †Biomaterials Innovation Research Center, Department of Medicine, Brigham and Women’s Hospital, Harvard Medical School, Cambridge, Massachusetts \\ 02139, United States \\ ${ }^{\ddagger}$ Harvard-MIT Division of Health Sciences and Technology, Massachusetts Institute of Technology, 77 Massachusetts Avenue, Cambridge, Massachusetts 02139, \\ United States \\ ${ }^{\S}$ Center for Biomaterials, Biomedical Research Institute, Korea Institute of Science and Technology, 14 Hwarang-ro, Seongbuk-gu, Seoul 02792, Republic of \\ Korea \\ "Department of Biomedical Engineering, ${ }^{\mathrm{I}}$ Department of Orthopedic Surgery, and ${ }^{\nabla}$ National Center for Regenerative Medicine, Case Western Reserve \\ University, Cleveland, Ohio 44106, United States \\ ${ }^{\perp}$ Department of Developmental BioEngineering, MIRA Institute for Biomedical Technology and Technical Medicine, and ${ }^{\#}$ Department of Biomechanical \\ Engineering, MIRA Institute for Biomedical Technology and Technical Medicine, University of Twente, Enschede 7522 NB, The Netherlands \\ ODepartment of Bioengineering, Department of Chemical and Biomolecular Engineering, Henry Samueli School of Engineering and Applied Sciences, University \\ of California-Los Angeles, Los Angeles, California 90095, United States \\ Department of Bioindustrial Technologies, College of Animal Bioscience and Technology, Konkuk University, Hwayang-dong, Gwangjin-gu, Seoul 143-701, \\ Republic of Korea \\ ${ }^{\dagger}$ Center of Nanotechnology, Department of Physics, King Abdulaziz University, Jeddah 21569, Saudi Arabia
}

\section{Supporting Information}

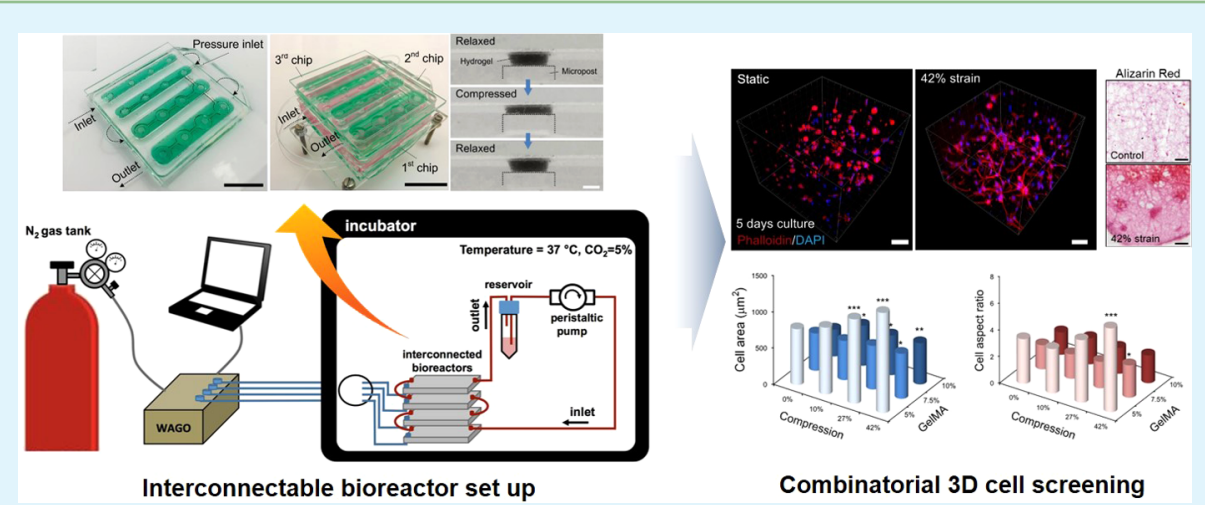

ABSTRACT: Biophysical cues can potently direct a cell's or tissue's behavior. Cells interpret their biophysical surroundings, such as matrix stiffness or dynamic mechanical stimulation, through mechanotransduction. However, our understanding of the various aspects of mechanotransduction has been limited by the lack of proper analysis platforms capable of screening three-dimensional (3D) cellular behaviors in response to biophysical cues. Here, we developed a dynamic compression bioreactor to study the combinational effects of biomaterial composition and dynamic mechanical compression on cellular behavior in 3D hydrogels. The bioreactor contained multiple actuating posts that could apply cyclic compressive strains ranging from 0 to $42 \%$ to arrays of cell-encapsulated hydrogels. The bioreactor could be interconnected with other compressive bioreactors, which enabled the combinatorial screenings of 3D cellular behaviors simultaneously. As an application of the screening platform, cell spreading, and osteogenic differentiation of human mesenchymal stem cells (hMSCs) were characterized in 3D gelatin methacryloyl (GelMA) hydrogels. Increasing hydrogel concentration from 5 to $10 \%$ restricted the cell spreading, however, dynamic compressive strain increased cell spreading. Osteogenic differentiation of hMSCs was also affected by dynamic compressive strains. hMSCs in 5\% GelMA hydrogel were more sensitive to strains, and the $42 \%$ strain group showed a significant increase in osteogenic differentiation compared to other groups. The interconnectable dynamic compression bioreactor provides an efficient way to study the interactions of cells and their physical microenvironments in three dimensions.

KEYWORDS: high-throughput screening, human mesenchymal stem cells, dynamic compression bioreactor, 3D mechanobiology, mechanical stimulation

\section{INTRODUCTION}

Cellular behaviors are continuously regulated by biochemical and biophysical stimuli. ${ }^{1-3}$ Biochemical stimuli, such as growth factors, have been shown to play a pivotal role in the development and regeneration of tissues and organs, ${ }^{4,5}$ and cell fate decisions can also be regulated by the stiffness of the

Received: November 25, 2017

Accepted: March 15, 2018

Published: March 15, 2018 
a

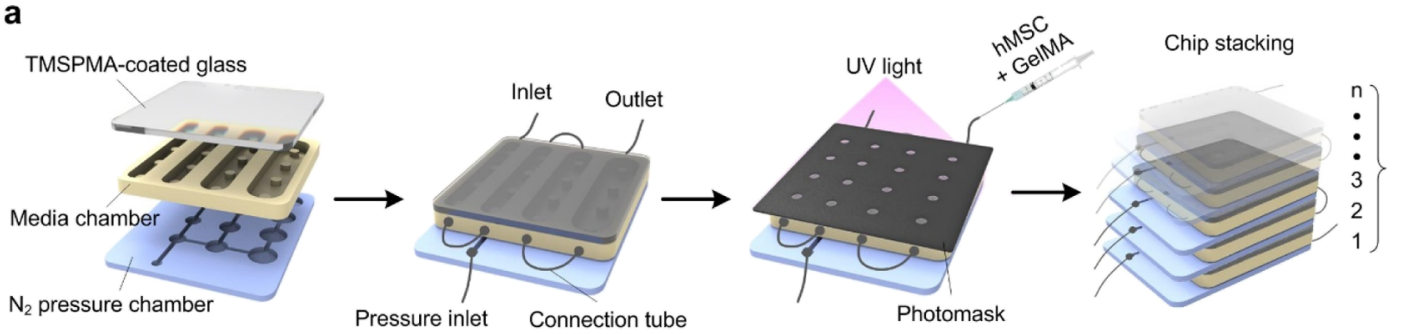

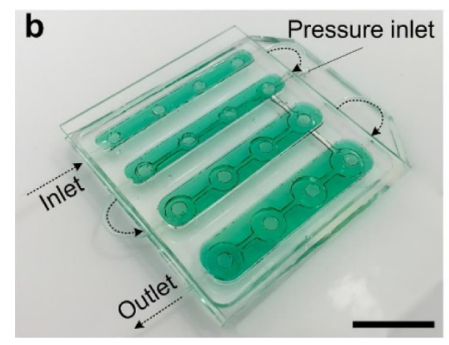

$$
\text { e }
$$

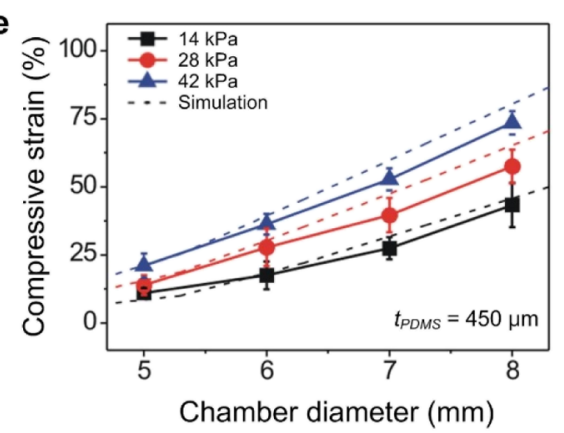

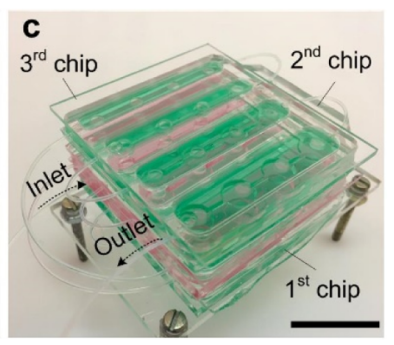
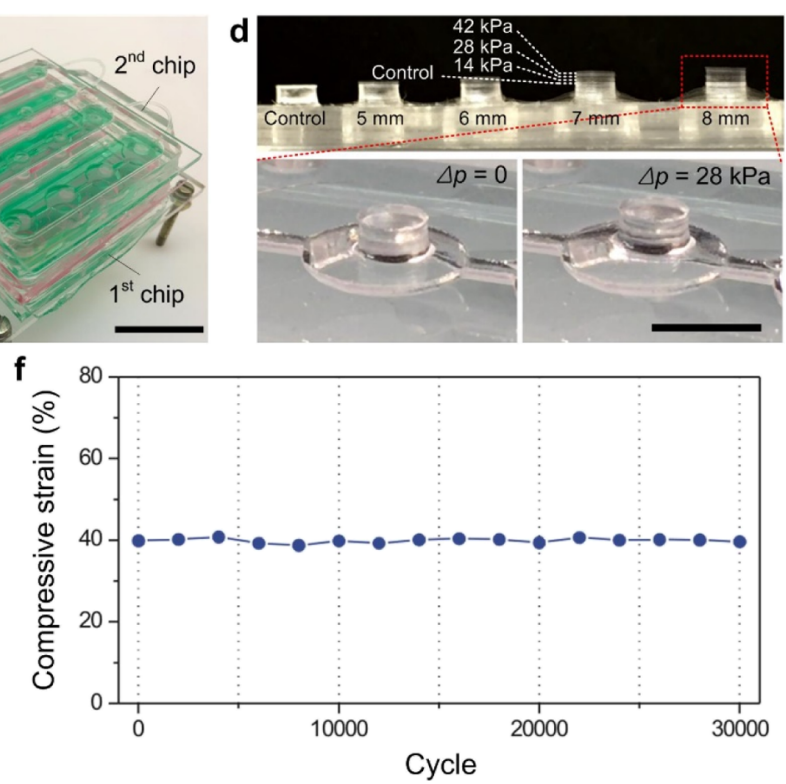

Figure 1. Dynamic compression bioreactors for the combinatorial screening of 3D cellular behaviors. (a) Schematic illustration of the interconnectable bioreactor fabrication process. Photographs of a (b) single and (c) interconnected bioreactor. Scale bar $=2 \mathrm{~cm}$. (d) Vertical displacement of posts depends on the pressure chamber diameter and amount of applied $\mathrm{N}_{2}$ pressure. Scale bar $=5 \mathrm{~mm}$. (e) Experimental and simulation data of compressive strains with $450 \mu \mathrm{m}$ PDMS membrane with different pressure chamber diameters and applied $\mathrm{N}_{2}$ pressures. (f) Stability test of the dynamic compression bioreactor over 30000 cycles of compression with $14 \mathrm{kPa} \mathrm{N}$ pressure and an $8 \mathrm{~mm}$ pressure chamber diameter.

extracellular matrix (ECM). ${ }^{6-8}$ In addition, cells can sense and respond to externally applied mechanical stimuli through mechanotransduction, which regulate cellular behaviors including cell adhesion, proliferation, and differentiation. ${ }^{9,10}$ A variety of biomaterials with tunable biochemical and physical properties have been developed to mimic microenvironments of native tissues. However, identifying microenvironmental conditions optimal for specific tissue engineering and regeneration applications through conventional approaches remains challenging. ${ }^{11-14}$ Recently, medium to high-throughput platforms have been introduced as promising strategies to screen the effects of biochemical and biophysical factors on cellular behaviors. ${ }^{15}$ It is possible for these platforms to contain arrays with distinct and isolated microenvironments, allowing simultaneous screening of a large number of conditions while requiring only a small amount of materials. Despite the advances in medium- to highthroughput technologies, current platforms have mostly focused on screening cellular behaviors in two-dimensional (2D) microenvironments under static culture conditions. ${ }^{16-19}$

Cellular responses in three-dimensional (3D) microenvironments are expected to more closely mimic responses in native tissues. $^{20-22}$ Therefore, arrays of 3D cell-laden biomaterials represent a powerful tool to assess the role of matrix properties and soluble biochemical signals on cellular behavior. In addition, externally applied mechanical stimuli, such as tension, compression, hydrostatic pressure, and shear stress, can affect 3D cell behaviors. For example, application of cyclic compression has been reported to promote cell differentiation ${ }^{23,24}$ and increase ECM synthesis ${ }^{25}$ in bone and cartilage tissue engineering approaches. The influence of mechanical stimulation on cell behavior is often a function of both matrix properties and the soluble biochemical milieu. ${ }^{26,27}$ However, the combinatorial effects of different dynamic compressive strains and biomaterial composition on stem cell differentiation in three dimensions are not fully understood, only few platforms have been developed to screen mechanical stimuli on 3D culture systems. ${ }^{28-33}$ Moraes et al. engineered a microfluidic screening platform that can apply different compressive strains to cell-laden poly(ethylene glycol) (PEG) hydrogel arrays. ${ }^{28}$ Recently, Liu et al. fabricated a deformable membrane platform to apply dynamic tensile strains to 3D human mesenchymal stromal cell-laden PEG hydrogel arrays. $^{29}$ Similarly, Li et al. reported a magnetically actuating hydrogel array platform to apply static tensile strains to cells cultured in 3D matrices. ${ }^{30}$ Although the aforementioned platforms could be used to screen $3 \mathrm{D}$ cell behaviors under different mechanical strains, the capability of a platform to screen the synergistic influences of different dynamic compressive strains and various biomaterial compositions under continuous perfusion of culture media has not been demonstrated.

In this paper, we have developed a bioreactor that can apply cyclic compressive strains to $3 \mathrm{D}$ cell-laden hydrogel arrays. Human mesenchymal stem cell (hMSC)-encapsulated gelatin methacryloyl (GelMA) hydrogel arrays were photocross-linked onto mechanically actuating posts, which can be operated by gas 
pressure. The actuation of posts enabled individual control over the compressive strains across each 3D hydrogel. The frequency and magnitude of compression could be precisely adjusted by changing applied gas pressure and device parameters of the bioreactor. Multiple bioreactors containing different hydrogel compositions could be connected to each other, which enabled combinatorial screening of 3D cellular behaviors in response to biomaterial and compressive strain combinations. As a proof of principle, cell spreading and osteogenic differentiation of hMSCs with different concentrations of GelMA and compressive strains were screened. Cell spreading and differentiation in three dimensions were investigated using cellular imaging, biochemical analysis, gene expression analysis, and histological analysis. The interconnectable dynamic compression bioreactor enabled the combinatorial screening of microenvironments with mechanical stimuli under continuous perfusion, which can be potentially used as an advanced screening platform for biomaterials and drug discovery applications.

\section{RESULTS AND DISCUSSION}

2.1. Fabrication and Characterization of Interconnectable Bioreactors. Interconnectable bioreactors for 3D combinatorial cell behavior screening were fabricated as illustrated in Figure 1a. The bioreactor was composed of three different layers: a nitrogen gas $\left(\mathrm{N}_{2}\right)$ pressure chamber, a media chamber, and a glass substrate. Polydimethylsiloxane (PDMS) was used to fabricate the media and $\mathrm{N}_{2}$ pressure chambers because of its excellent flexibility and biocompatibility. ${ }^{34,35}$ The $\mathrm{N}_{2}$ pressure chamber layer was composed of cylindrical chambers connected by channels to apply $\mathrm{N}_{2}$ pressure through a single pressure inlet. The media chamber was composed of posts and sidewalls on a thin PDMS membrane. The heights of sidewall and post were 3 and $1.5 \mathrm{~mm}$, respectively. The bioreactor was assembled by plasma-bonding the two PDMS layers and a glass substrate together, which was followed by the incorporation of polytetrafluoroethylene (PTFE) connectors. Here, a 3(trimethoxysilyl)propyl methacrylate (TMSPMA)-treated glass was used to provide long-term adhesion stability of the hydrogel arrays during dynamic compression. ${ }^{36}$ To pattern the hydrogel array, GelMA prepolymer solution with hMSCs was injected into the media chamber and selectively photocross-linked onto the posts by using UV light and a photomask. Notably, the uncrosslinked prepolymer solution could be removed from the device and re-injected into other bioreactors; this effectively minimized material consumption. A single bioreactor contained 16 samples, and a stack of bioreactors could be interconnected for the combinatorial screening of 3D cellular behaviors (Figure 1b,c). When the gas pressure was applied through the $\mathrm{N}_{2}$ pressure chambers, the PDMS membrane with posts was deflected upward because of membrane stretching. This allowed for the vertical displacement of the posts to apply compressive strains across cell-laden hydrogel arrays. Figure 1d shows photographs of actuating posts with different applied gas pressures (14-42 $\mathrm{kPa})$ and chamber diameters $(5-8 \mathrm{~mm})$. The displacement of the posts could be modulated by changing the $\mathrm{N}_{2}$ pressure chamber diameter and the applied gas pressure. To determine the displacement of actuating posts when gas pressure was applied, the displacements of posts were measured from the side view by varying the PDMS membrane diameter and applied gas pressure. The compressive strain, the ratio between the displaced distance of the post and the initial distance between the glass and post, is presented in percent to indicate how much compressive strain can be applied to the hydrogel. Figure le shows the relationship between the compressive strain and the $\mathrm{N}_{2}$ chamber diameter at different applied pressures. It was observed that the compressive strains positively correlated with applied pressure and chamber diameter. The compressive strain was also affected by the thickness of the PDMS membrane $\left(t_{\mathrm{PDMS}}\right)$ (Figure $\left.\mathrm{S} 1\right)$. A numerical simulation was carried out to compare the empirical results with theoretical values. The simulation data were obtained for travel distances of the top post surface with the applied pressures of 14,28 , and $42 \mathrm{kPa}$. The experimental results were in good agreement with the numerical simulation model data, with small differences attributed to variations of $t_{\mathrm{PDMS}}$ during the fabrication of the bioreactors and misalignments between posts and gas pressure chambers. The bioreactor's post displacement was highly reproducible and stable over 30000 compressive cycles (Figure 1f; Movie S1). By changing the device parameters and applied pressure, post displacement could be accurately and reproducibly controlled from 0 to nearly $90 \%$. Thus, the dynamic compression bioreactor could potentially be used to screen the 3D cellular behaviors over a large range of physiological and pathophysiological strains. ${ }^{37,38}$ This capability could advance mechanobiological investigations in the areas of musculoskeletal tissue development, remodeling, and repair. ${ }^{39-42}$ In the following experiments, $t_{\mathrm{PDMS}}=450 \mu \mathrm{m}$ with $14 \mathrm{kPa}$ of the applied pressure was used, which enabled the displacement of posts from $0.15 \pm 0.03$ to $0.63 \pm 0.12 \mathrm{~mm}$ when varying the diameter of the pressure chamber $(5-8 \mathrm{~mm})$. This in turn resulted in $10.5 \pm 1.8$ to $42 \pm 8.1 \%$ compression of hydrogels located between the posts and glass.

\subsection{Mechanical Properties of GelMA Hydrogels.} Cellular behaviors are directly regulated by the cells' microenvironment. Importantly, biomaterial mechanical properties and active mechanical stimulation have been shown to affect cellular behaviors in diverse ways. ${ }^{6,7}$ To screen the combinational effects of biomaterial composition and dynamic compressive strain on hMSC behaviors in three dimensions, three different concentrations of GelMA hydrogels $(5,7.5$, and $10 \%$, w/v) were used. To measure mechanical properties of the GelMA hydrogels, GelMAs were cross-linked, swollen in Dulbecco's phosphate buffered saline (DPBS), and punched into cylindrical samples (10 mm diameter and $1 \mathrm{~mm}$ height). The compressive Young's moduli of GelMA hydrogels were measured by determining the slope of the elastic region of stress-strain curves. The measured compressive Young's moduli were positively correlated with the hydrogel concentrations (Figure $2 \mathrm{a}, \mathrm{b})$. The Young's moduli of 5, 7.5, and 10\% GelMA hydrogels were 5, 19, and $29 \mathrm{kPa}$, respectively. Although the GelMA hydrogels showed pronounced hysteresis, all hydrogels fully recovered their original thickness after unloading (Figure 2c). Figure $2 \mathrm{~d}$ and Movie S2 show the cyclic deformation of $5 \%$ GelMA hydrogel under $42 \%$ compressive strains. The patterned hydrogels have a tapered shape, which may be attributed to the UV light deflections from the edges of the photomask during the photocross-linking process. ${ }^{43}$ When gas pressure is applied, the PDMS membrane with post deflects upward, which uniformly compresses the hydrogel between the post and the glass slide. The applied strains onto the hydrogels through the HT device were decreased $(38 \%)$ than the value without hydrogel (42\%), which is attributed to the presence of hydrogel and media in the chamber. However, the strain differences related to the presence of hydrogels were less than $6 \%$ for all the conditions, and there were no significant differences of the compressive strains between the HT device with and without hydrogels. Figure $2 \mathrm{e}, \mathrm{f}$ shows the dynamic compression responses of the patterned 

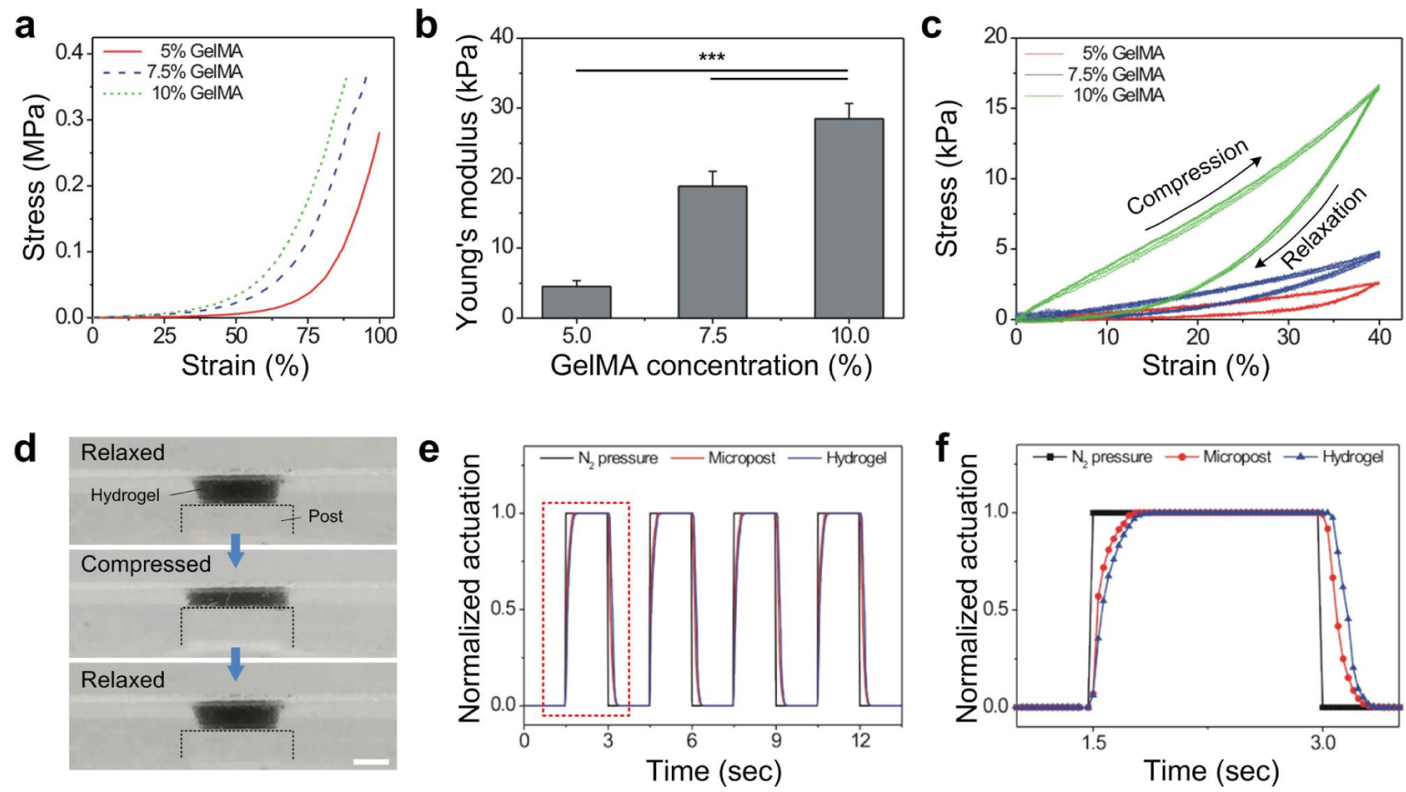

Figure 2. Mechanical properties of the GelMA hydrogel. (a) Representative stress-strain curves from compression tests, (b) Young's modulus of GelMA hydrogels, and (c) cyclic compression test (10 cycles) of GelMA hydrogels with different hydrogel concentrations (5, 7.5, and 10\%). (d) Sequential photographs showing the compression of a patterned hydrogel under compression. Black dye was incorporated to the hydrogel to aid in visualization. Scale bar $=1 \mathrm{~mm}$. (e,f) Characterization of the dynamic compression response of a patterned hydrogel upon actuation of post by applied pressure.
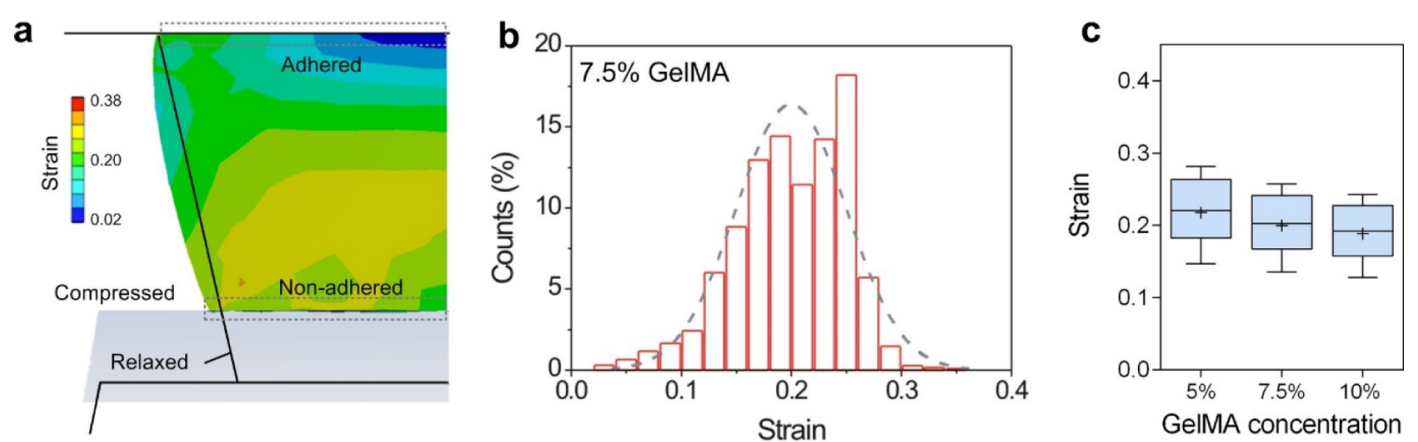

Figure 3. (a) Numerical simulation of the strain distribution within the hydrogel. (b) Statistical analysis of strain distribution of the $7.5 \%$ GelMA hydrogel and (c) effect of the hydrogel concentration on overall strain distributions in compressed hydrogels.

hydrogel upon the actuation of a post with a frequency of $0.3 \mathrm{~Hz}$. The compression cycle was programmable, and the displacement of the post and 3D hydrogel tightly and rapidly followed the applied gas pressure. The results indicate that the GelMA hydrogel can be used as a biomaterial for the screening of $3 \mathrm{D}$ cellular behaviors under repetitive dynamic compressive strains.

2.3. Numerical Simulation to Characterize Dynamic Compression of the Hydrogel. Computational modeling was conducted to predict the vertical strain distribution within the hydrogel during its compression (Figure 3a). The strain model of hydrogel compression was based on a pressure chamber diameter of $6 \mathrm{~mm}, t_{\mathrm{PDMS}}$ of $450 \mu \mathrm{m}$, and a gas pressure of $14 \mathrm{kPa}$. The strain distribution within the hydrogel sample showed that the compressive strain was not equally distributed throughout the hydrogel volume. The hydrogel experienced the largest strain near its bottom surface where it could freely move over the post surface, while the strain was lowest near the top of the hydrogel where it was fixed to the glass. The hydrogel fixation to the glass prevented local lateral expansion of the hydrogel, which in turn, limited the local deformation in the vertical direction. The statistical strain distributions within 7.5\% GelMA hydrogels under compressive strains were analyzed as shown in Figure 3b,c. The simulation data indicated that the strain distribution in the hydrogel tended to follow a normal distribution for all GelMA hydrogel concentrations under compressive strains. In addition, it was found that the presence of hydrogel samples in the bioreactor only had a marginal effect on the displacement of the post. The displacement distance of the post slightly dropped from $0.33 \mathrm{~mm}$ without a hydrogel to $0.32,0.31$, and $0.30 \mathrm{~mm}$ when GelMA hydrogels of 5, 7.5, and $10 \%$ were loaded, respectively. We observed that there were small decreases of applied strains when the hydrogels were patterned into the HT device. However, no significant differences of applied compressive strains were observed between GelMA hydrogel concentrations under the same gas pressure. Consequently, the effects of the hydrogel concentration on the compressive strain during the compression were minimal because of the relatively low Young's modulus of all hydrogel compositions compared to PDMS (1-4 $\mathrm{MPa})$ and glass $(50-90 \mathrm{GPa}){ }^{44}$ It should be noted that the computational model did not include cells embedded in the hydrogel compartment. As a result, the actual strain values may be different from the predicted distributions. However, differ- 
ences are expected to be small unless the cellular density becomes extremely high, because the Young's modulus and Poisson's ratio of cells are in the same range as the properties used for the hydrogel in this study. ${ }^{45}$

2.4. Cell Compatibility of the Bioreactor and Experimental Setup. To demonstrate the feasibility of combinatorial screening of $3 \mathrm{D}$ hMSC behaviors, the effects of different combinations of GelMA hydrogel concentrations (5, 7.5, and $10 \%)$ and dynamic compressions $(0,10,27$, and $42 \%)$ on cell viability and spreading behavior were examined using multiple interconnected bioreactors. The screening platform's experimental setup consisted of interconnected bioreactors, a gas pressure applying module, a media reservoir, and a peristaltic pump (Figure $4 \mathrm{a}$ ). The screening platform was designed to continuously perfuse media from the reservoir during cell culture to supply cells with sufficient nutrients and oxygen, while simultaneously removing metabolic byproducts and waste. The gas pressure module was located outside the incubator and connected to the bioreactors via tubing. Gas pressure was applied to each bioreactor $12 \mathrm{~h}$ post cell seeding, and cyclic pressure was programmed by using multiple solenoid valves controlled by a MATLAB software tool and a WAGO controller. To test the cell viability of the screening platform, hMSCs $\left(1 \times 10^{6}\right.$ cells $\left./ \mathrm{mL}\right)$ were encapsulated in 5\% GelMA hydrogels and cultured for 7 days. Cell viability studies were performed under various dynamic compression regimes (0-42\%) using a Live/Dead staining kit on days $0,3,5$, and 7 . For the cell viability study, 0.3 $\mathrm{Hz}$ compressive strain (1.5 s compression and $1.5 \mathrm{~s}$ relaxation time) was continuously applied to the hMSC encapsulated hydrogels during the cell culture. Figure $4 \mathrm{~b}$ shows representative images of Live/Dead stained cells in hydrogels under static conditions, which demonstrate the biocompatibility of the bioreactor under continuous perfusion of cell culture media. Small decreases of cell viability were observed under 27 and $42 \%$ compressive strains after 5 days, but high cell viability of $>80 \%$ was observed for all conditions. The results indicate that the photocross-linking process and dynamic mechanical compression had minimal effect on hMSC survival.

2.5. Screening of 3D Cellular Behaviors Using the Interconnectable Bioreactor. Combinatorial screening of 3D hMSC behaviors was performed by using the developed screening platform. hMSCs were cultured for 5 days with continuous dynamic compression at a rate of $0.3 \mathrm{~Hz}(1.5 \mathrm{~s}$ compression and $1.5 \mathrm{~s}$ relaxation time). hMSC spreading was affected by both hydrogel concentration and compressive strain magnitude. Representative fluorescence microscope images (Figure $5 \mathrm{a}-\mathrm{c}$ ) depict the representative hMSC spreading behavior in three different GelMA hydrogel concentrations without dynamic compression. Cell area and aspect ratio decreased as the GelMA hydrogel concentration increased. The reduced spreading and growth of cells in higher GelMA hydrogel concentrations may have resulted from the smaller pore size of the polymer networks as the degree of cross-linking increases in these hydrogels. From scanning electron microscopy (SEM) images of lyophilized GelMA hydrogels at different concentrations, the hydrogel network was denser and the average pore size was significantly smaller in 10\% GelMA hydrogels (1.91 $\pm 0.81 \mu \mathrm{m})$ than in those of $5 \%$ hydrogels $(18.24 \pm 4.52 \mu \mathrm{m})$ (Figure 5d). Similar restrictions of cell spreading and growth in dense hydrogel networks were observed in other biomaterials including PEG-GelMA hydrogel mixtures. ${ }^{46,47}$ In addition to the pore size of hydrogels, the density of cell binding sites can affect cell spreading behavior. Since GelMA hydrogels contain

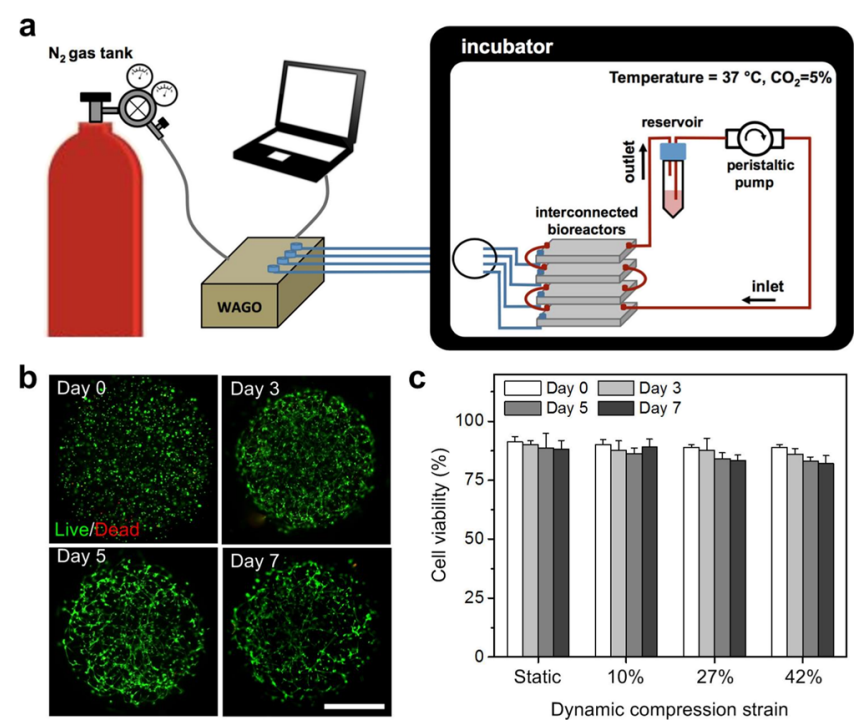

Figure 4. (a) Schematic representation of the experimental setup of interconnected dynamic compression bioreactors for screening 3D cellular behaviors. (b) Live/Dead staining of hMSCs in 5\% GelMA hydrogel without compression. Scale bar $=1 \mathrm{~mm}$. (c) Cell viability with different dynamic compression magnitudes over time $(N=4)$.

cell adhesion binding domains, it is not possible to independently control hydrogel cell adhesivity and pore size when changing macromer concentration. ${ }^{47}$ This would be possible by using GelMA with different levels of methacrylation, and future studies could be performed to determine the independent and synergistic effects of cell binding sites and hydrogel stiffness on cell behavior in this system.

Not only did the polymer network of the hydrogels affected cell spreading, but dynamic compression of the hydrogels did as well. 3D reconstructed confocal images of hMSCs in 5\% GelMA hydrogels or static conditions clearly revealed that cyclical $42 \%$ strain substantially increased cell spreading (Figure 5e). The confocal images were taken from the bottom part of hydrogel to exclude the cells near the glass substrate where strains were not effectively applied. The combinational effects of dynamic compression and hydrogel concentration on cell spreading were then investigated. For all hydrogel concentrations, cell spreading increased in response to increased dynamic compressive strain (Figures 5f and S2). This response was the strongest in the 5\% GelMA hydrogel group. Similar 3D cell spreading behavior with dynamic cyclic compression mechanical stimulation was observed for NIH-3T3 fibroblasts and human mesenchymal stromal cells in previous reports. ${ }^{29,30}$

2.6. Screening of Osteogenic Differentiation of hMSCs in Three Dimensions. The correlation between microenvironment factors and stem cell differentiation has been actively investigated and screened for several years but mostly in 2D environments. ${ }^{48,49} 2 \mathrm{D}$ environments are simpler to establish, but $2 \mathrm{D}$ systems by their nature are not sufficient to represent or simulate the $3 \mathrm{D}$ cellular environments of tissues in the body. ${ }^{50,51}$ Moreover, native tissues and cells are exposed to different external stimulations including tension, shear strain, compression, and so forth, which can regulate in cell and tissue functions. ${ }^{52,53}$ Many strategies to drive stem cell differentiation have utilized the application of external stress to simulate physiologically relevant mechanical environments present during tissue formation, repair, and homeostasis, ${ }^{54-57}$ but there are limited self-contained systems with the capacity to examine the 

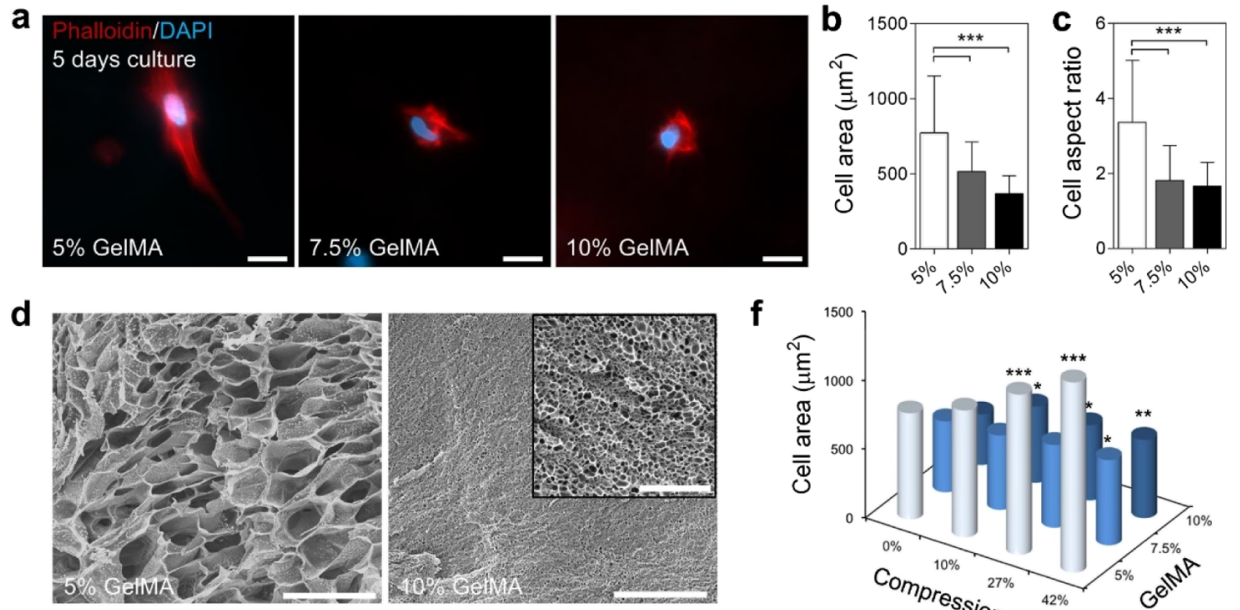

f
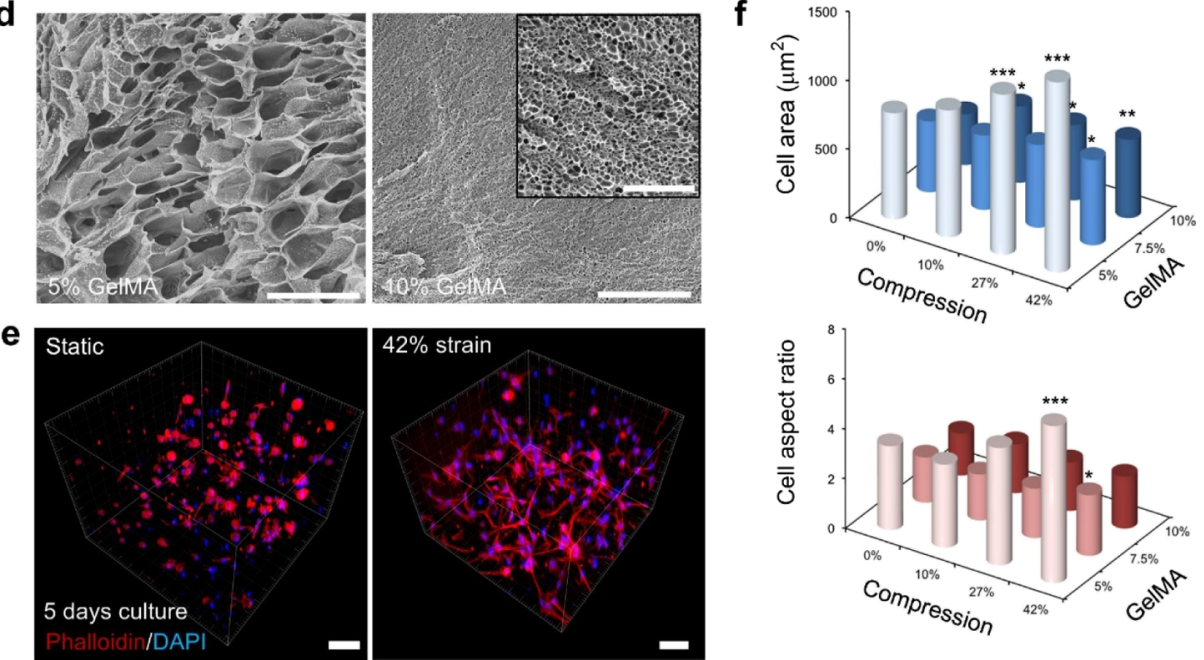

Figure 5. Combinatorial screening of hMSC cellular morphology after 5 days of culture. (a) Representative photomicrophotographs of hMSCs in GelMA hydrogels composed of different concentrations of polymer without compression. F-Actin and nucleus of hMSCs were stained with phalloidin and DAPI, respectively. Scale bar $=20 \mu \mathrm{m}$. (b) Cell area and (c) cell aspect ratio for each hydrogel made with different polymer concentrations $(N>30$ cells for each condition, ***p $<0.001$ compare to the group of $5 \%$ GelMA hydrogel). (d) Typical SEM images of the lyophilized hydrogel network. Scale bar $=50 \mu \mathrm{m}$. Inset shows a higher magnification of the SEM image of 10\% GelMA hydrogel. Scale bar $=10 \mu \mathrm{m}$. (e) 3D reconstructed confocal photomicrophotographs of hMSCs in 5\% GelMA hydrogel cultured under different strains. Scale bar $=100 \mu \mathrm{m}$. (f) Combinatorial screening of effect of hydrogel composition and dynamic compression on hMSC morphology $(N>30$ cells for each condition, $* * * p<0.001, * * p<0.01, * p<0.5)$.

role of such mechanical signals on cell differentiation in conjunction with multiple different biomaterial compositions. Therefore, we examined osteogenic differentiation of hMSCs encapsulated in 3D GelMA hydrogels with different macromer concentrations under varied compressive stimulation regimes. As a preliminary screen, osteogenic differentiation was investigated as a function of GelMA concentration and compressive strain magnitude at day 7 (Figure 6a). Different strains at $0.3 \mathrm{~Hz}$ compressive strain were applied to the hydrogels for $3 \mathrm{~h} /$ day for the 7 days of culture. Similar to the cell spreading behavior (Figure 5), alkaline phosphatase (ALP) activity, an early stage osteogenic marker, ${ }^{58}$ increased with increased compressive strain in 5\% GelMA hydrogel. However, there was no significant effect of compression on ALP activity in 7.5 and 10\% GelMA hydrogel groups. The low ALP activities in 7.5 and 10\% GelMA could be attributed to the dense pore structure and low degradability of GelMA hydrogel, which may disturb cell spreading and dynamic mechanotransduction of hMSCs. ${ }^{33}$ On the basis of the ALP screening results, hMSCs encapsulated 5\% GelMA hydrogels were selected as a promising condition in which the cells were responsive to compressive mechanical stimulation, and these constructs were subjected to a range of compressive strains over time (Figure $6 \mathrm{~b}-\mathrm{e}$ ). Runt-related transcription factor 2 (Runx2), another important gene early in the osteogenic differentiation pathway of progenitor cells, can be upregulated by stretching stimulation in $2 \mathrm{D}$ and $3 \mathrm{D}$ environments. ${ }^{59,60}$ Therefore, the gene expression of mechanosensitive Runx 2 in hMSCs was examined at day 7. Runx2 gene expression in the $42 \%$ strain group was significantly higher than that of the 0,10 , and $27 \%$ strain groups
(Figure 6b). ALP activity of the constructs increased with increasing compressive strain magnitude at days 7 and 21 . Although the average ALP activity increased over time for the stimulated groups, significant increases were found in the $27 \%$ (day 7 ) and $42 \%$ (days 7 and 21 ) compression groups (Figure 6c). After 21 days, late markers of osteogenesis, osteocalcin (OCN), osteopontin (OPN), and calcium, stained more intensely in the $42 \%$ strain group than the $0 \%$ strain group, further confirming enhanced osteogenic differentiation (Figure $6 \mathrm{~d}, \mathrm{e})$. The OCN, OPN, and calcium staining intensities of strain 10 and $27 \%$ groups were similar to the $0 \%$ strain group (data not shown). Corroborating the alizarin red S staining, quantification of calcium deposition demonstrated significantly higher amounts in the $42 \%$ strain group compared to all other groups at 21 days, while there was no significant difference among the lower strain conditions. During osteogenic differentiation, there was a 36$42 \%$ decrease in average DNA content from day 7 to day 21 within the same groups, but there was no significant difference among the different strains (Figure S3). The overall DNA content decrease may have resulted from two factors; cell washout and the osteogenic differentiation. Some encapsulated cells may have come out of the gels because of the movement of media within the reactor. Second, previous reports have found decreased cell viability with increased osteogenic differentiation $^{61-63}$ and that could have occurred in this system. Because there was a similar DNA content among the groups, compressive strain does not appear to have affected the cell viability. Overall, hMSCs encapsulated in the 5\% GelMA hydrogels with $42 \%$ applied compressive strain resulted in the best osteogenic 
a

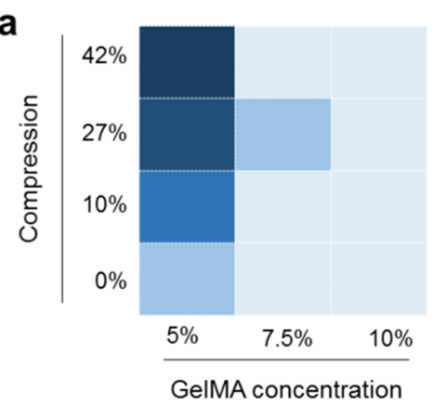

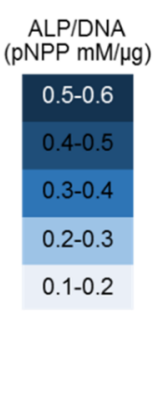

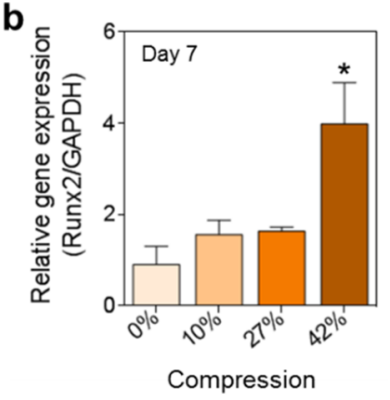

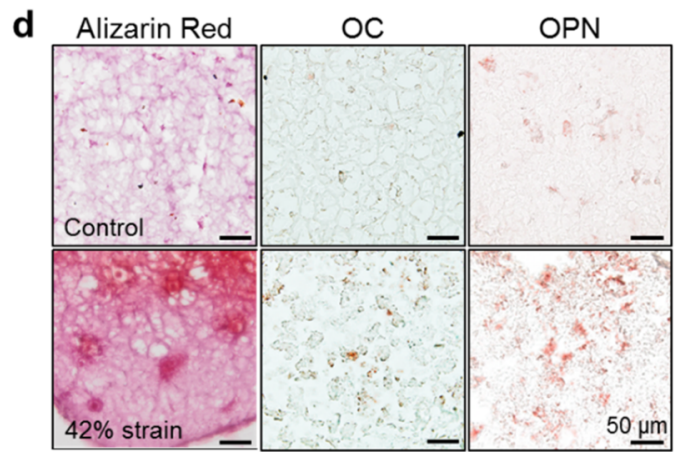

e

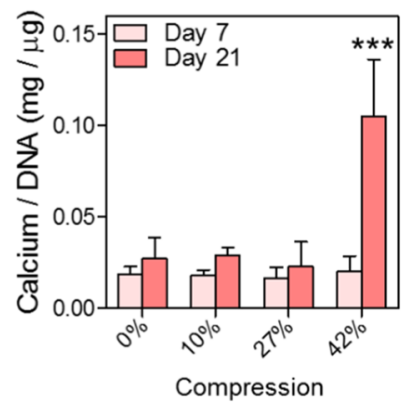

Figure 6. Combinatorial screening of osteogenic differentiation of hMSCs encapsulated in GelMA hydrogels. (a) ALP expression of encapsulated hMSCs in different GelMA hydrogel compositions and compressive strains after 7 days. (b) Relative mRNA levels of Runx2 of hMSCs encapsulated in $5 \%$ GelMA under different compressive strains after 7 days of differentiation ( ${ }^{*} p<0.05$ compared to the $0 \%$ strain group). (c) ALP expressions of hMSCs encapsulated in 5\% GelMA hydrogels $(\mathrm{w} / \mathrm{v})$ with various compressive strains after 7 and 21 days of osteogenic differentiation $(* p<0.05$ compared to the $0 \%$ strain group at day 7 and $* * p<0.05$ compared to the $0 \%$ strain group at day 21 ). (d) Representative photomicrographs of alizarin red S, OCN, and OPN staining after 21 days of osteogenic differentiation. Scale bars $=50 \mu \mathrm{m}$. (e) Quantitative calcium deposition analysis of hMSCs at days 7 and $21(* * * p<0.05$ compared to all of the other groups at day 21$)$.

differentiation among the conditions studied. This result may suggest that cells in a $3 \mathrm{D}$ environment can display better osteogenic differentiation when they are exposed to significant compressive strains within porous hydrogels. These combinational approaches will help to find the better cellular environments that can be applied for the therapeutic bone regenerations.

\section{CONCLUSIONS}

We have developed an interconnectable bioreactor that can screen combinational effects of dynamic compression and hydrogel compositions on hMSCs in a high-throughput manner. Specifically, the interconnectable characteristic of the developed bioreactor setup enables high-throughput combinatorial screening of the role of insoluble (e.g., compressive stress, hydrogel stiffness, and biochemical properties, etc.) and soluble (e.g., media composition, growth factors, etc.) microenvironmental parameters on cell behavior. As a proof-of-concept, 3D hMSC behaviors including spreading and osteogenic differentiation in different concentrations of GelMA hydrogels were screened using the developed bioreactor. When compressive strain was applied to different concentrations of GelMA hydrogels, encapsulated hMSCs were affected by dynamic compression and resulted in enhanced cell spreading and osteogenic differentiation. In the 5\% GelMA group, higher magnitudes of dynamic compression significantly increased cell spreading and osteogenic differentiation. Further investigation of hMSC functions such as viability, mechanotransduction, and intercellular signaling with this screening platform will pave the way for understanding the role of biomaterial properties in concert with mechanical stimulation on cell behavior. In addition, the developed bioreactor setup can be used to investigate other in vitro organ models, particularly mechanically active tissues such as cartilage, tendons, muscles, and blood vessels. These in vitro organs-on-a-chip models can be further connected together through the microfluidics in a similar manner to how they are arranged in vivo, providing the potential capability to study interactions between in vivo-like tissue models. To control and monitor dynamic changes in the bioreactor culture microenvironment, the development of non-invasive, in situ sensors for $\mathrm{O}_{2}$ level, $\mathrm{pH}$, and $\mathrm{CO}_{2}$ level would be valuable. In addition to GelMA hydrogel, the system is compatible with a wide range of other hydrogel compositions, and it may also be used for drug screening and toxicology applications.

\section{EXPERIMENTAL SECTION}

4.1. Synthesis of GeIMA. GelMA was synthesized as described previously. ${ }^{64}$ Briefly, $10 \mathrm{~g}$ of type A porcine skin gelatin (Sigma) was fully dissolved into $100 \mathrm{~mL}$ of DPBS (Sigma) at $60{ }^{\circ} \mathrm{C}$. Methacrylic anhydride ( $8 \mathrm{~mL}$; Sigma) was added dropwise to the solution and stirred magnetically at $50^{\circ} \mathrm{C}$ for $2 \mathrm{~h}$. The solution was then diluted with DPBS to stop the methacrylation reaction. The unreacted methacrylic anhydride and salts were removed through 7 days of dialysis in $50{ }^{\circ} \mathrm{C}$ distilled water using $12-14 \mathrm{kDa}$ cut-off dialysis tubes. The dialyzed solution was frozen at $-80{ }^{\circ} \mathrm{C}$ and then lyophilized for 5 days. Lyophilized GelMA was kept at $-80^{\circ} \mathrm{C}$ before use.

4.2. Characterization of the GelMA Hydrogels. Lyophilized GelMA was dissolved in cell culture medium with $0.25 \%(\mathrm{w} / \mathrm{v})$ photoinitiator (Irgacure 2959; Ciba Specialty Chemicals; Tarrytown, NY, USA) at $80^{\circ} \mathrm{C}$ for $1 \mathrm{~h}$ to prepare GelMA prepolymer solution. To mechanically characterize the GelMA hydrogels, three different macromer concentrations (i.e., 5, 7.5, and $10 \mathrm{w} / \mathrm{v} \%$ ) were prepared, and $1 \mathrm{~mL}$ of each GelMA prepolymer solution was placed on a glass plate with two $1 \mathrm{~mm}$-thick spacers. The prepolymer solution was then covered with a quartz plate and cross-linked by using $25 \mathrm{~mW} / \mathrm{cm}^{2} \mathrm{UV}$ 
light for $30 \mathrm{~s}$. Then, the cross-linked hydrogel was punched into $10 \mathrm{~mm}$ diameter disks. Compression tests were performed using a mechanical testing machine ( $1 \mathrm{kN}$ Actuator, TestResources, Shakopee, MN, USA) equipped with a $5 \mathrm{~N}$ load cell, and the $30 \%$ compressive strain was applied to the hydrogels at a constant crosshead speed of $1 \%$ strain $/ \mathrm{s}(\mathrm{N}$ $=4$ ). The value of Young's modulus was calculated by measuring the first $5 \%$ of the stress-strain curve where there was a nonzero stress. ${ }^{65}$ For the cyclic compression test, a constant crosshead speed of $1 \%$ strain/s was applied, and 10 loading/unloading cycles with $40 \%$ compressive strain were applied to the hydrogels $(N=5)$. Surface morphologies of the GelMA hydrogels were characterized using a field emission scanning electron microscope (Hitachi S4700; Hitachi, Tokyo, Japan). Before the SEM imaging, the hydrogel samples were freeze-dried and coated with a thin Pt layer using a SEM sputter coater for $30 \mathrm{~s}$ (a pressure of $\approx 0.3 \mathrm{~atm}$ with an anode current of $40 \mathrm{~mA}$ ).

4.3. hMSC Isolation and Culture. Whole bone marrow was harvested from the iliac crest of healthy patients at the Case Comprehensive Cancer Center Hematopoietic Biorepository and Cellular Therapy Core with approval from University Hospitals of Cleveland Institutional Review Board. hMSCs were isolated from the marrow via a Percoll (Sigma) gradient and the differential cell adhesion method. ${ }^{66}$ Only the adherent hMSCs were collected and cultured in Dulbecco's modified Eagle's medium-low glucose (DMEM-LG, Sigma) supplemented with $10 \%$ fetal bovine serum (FBS, Sigma), $1 \%$ penicillin streptomycin (PS, Invitrogen, Carlsbad, CA, USA), and 10 $\mathrm{ng} / \mathrm{mL}$ recombinant human fibroblast growth factor-2 (rh-FGF-2; R\&D Systems). To osteogenically differentiate hMSCs, cells were cultured in DMEM-high glucose (DMEM-HG, Sigma) supplemented with 10\% FBS, $1 \%$ PS, $10 \mathrm{mM} \beta$-glycerophosphate (Calbiochem, Billerica, MA), $50 \mathrm{mM}$ ascorbic acid (Wako USA, Richmond, VA), and $100 \mathrm{nM}$ dexamethasone (MP Biomedicals, Solon, $\mathrm{OH}$ ). Passage 3 hMSCs were used in the experiments.

4.4. Fabrication of the Dynamic Compression Bioreactor. Two poly(methyl methacrylate) (PMMA) molds to form the PDMS $\mathrm{N}_{2}$ pressure chamber and media chamber were obtained using a laser cutter. The mold for the $\mathrm{N}_{2}$ pressure chamber layer composed of the inverse of cylindrical chambers with different diameters (i.e., 5, 6, and $8 \mathrm{~mm}$ ) that were all connected via $1.5 \mathrm{~mm}$ wide connecting channels to enable the application of pressure from a single gas inlet. The media chamber was patterned with the inverse of a $4 \times 4$ array of posts ( $3 \mathrm{~mm}$ in diameter) and sidewalls. The height of the sidewalls ( $3 \mathrm{~mm}$ in height) was designed to be higher than the posts ( $1.5 \mathrm{~mm}$ in height) to pattern the $3 \mathrm{D}$ hydrogel arrays between the posts and the upper glass substrate. The media chamber dimension needed to be designed larger than the $\mathrm{N}_{2}$ pressure chamber. The corresponding PDMS layers were fabricated by casting a 10:1 w/w mixture of PDMS base and curing agent (Sylgard 184; Dow Corning, MI, USA) onto the PMMA molds and curing at 80 ${ }^{\circ} \mathrm{C}$ for $1 \mathrm{~h}$. To obtain the desired membrane thickness of the media chamber layer, multiple adhesive tapes were attached to the side of the mold as spacers with different heights. PDMS was poured onto the PMMA mold with a spacer, and redundant PDMS was squeezed out from the mold using a flat PMMA substrate. The thickness of the cured PDMS could be controlled by varying the thickness of the spacer (Figure S4). The spacers with thicknesses 230,310 , and $400 \mu \mathrm{m}$ were used to obtain $\sim 300, \sim 450$, and $\sim 560 \mu \mathrm{m}$ thick PDMS membranes, respectively. Both layers were peeled off from the molds and plasmabonded. Upon bonding of the PDMS layers, fluidic and gas ports were cored using a needle. The PDMS structure was then plasma-bonded with a TMSPMA-coated glass. ${ }^{36}$ PTFE tubes (Microbore PTFE tubing, Cole-Palmer, Veron Hills, IL, USA) were used to connect inlet and outlet ports.

4.5. Cell Encapsulation and Culture in the Bioreactor. hMSCs were trypsinized from the flask and suspended in GelMA prepolymer solutions at a cell density of $1 \times 10^{6}$ cells $/ \mathrm{mL}$ (cell spreading and viability study) or $1 \times 10^{7}$ cells $/ \mathrm{mL}$ (osteogenic differentiation study). The cell-suspended GelMA prepolymer solutions were injected into the media chamber of the bioreactor through the connection tube. A photomask (circular shapes, $3 \mathrm{~mm}$ diameter) was then manually aligned on the top of the bioreactor to selectively polymerize the hydrogel onto the post array. The cell containing prepolymer solution was subsequently cross-linked by UV light exposure $\left(25 \mathrm{~mW} / \mathrm{cm}^{2}\right)$ for 30 s. The uncross-linked prepolymer solution was removed, and the chamber was filled with normal hMSC culture media with $10 \mathrm{ng} / \mathrm{mL}$ of rh-FGF-2 or osteogenic differentiation media. The bioreactors containing hydrogel array formed with different macromer concentrations were interconnected with each other and were perfused at a flow rate of $100 \mu \mathrm{L} / \mathrm{min}$ using a single peristaltic pump. All cell culture experiments were performed in a humidified incubator with $5 \% \mathrm{CO}_{2}$ at $37^{\circ} \mathrm{C}$. To apply cyclic compressions to the cross-linked hydrogel within the bioreactor, $\mathrm{N}_{2}$ gas pressure was supplied through the pressure inlet by using multiple solenoid valves that were controlled by a WAGO controller and a custom-designed MATLAB program. $\mathrm{N}_{2}$ was used as the working gas to operate the dynamic compressive bioreactor as it has been reported in the literature to operate pneumatic actuators for microfluidic devices and bioreactors without affecting cell behaviors. ${ }^{29,34}$ For the cell spreading study, $0.3 \mathrm{~Hz}$ compressive strain was continuously applied to the hMSC-encapsulated hydrogels during 5 days of culture. For the osteogenic differentiation study, $0.3 \mathrm{~Hz}$ compressive strain was applied to the hydrogels for $3 \mathrm{~h} /$ day during the 21 days of culture.

4.6. Computational Simulation. Computational finite element models were developed using ANSYS 13.0 Workbench to analyze the deformation of the PDMS membranes and the subsequent strain distribution within the hydrogel samples. A quarter unit of the hydrogel was modeled with symmetry boundary conditions. The models consisted of three parts: the PDMS structure matching the dimensions of the actual devices, the hydrogel sample modeled as a tapered cylinder with a height of $1.5 \mathrm{~mm}$, a top diameter of $2.5 \mathrm{~mm}$ and a bottom diameter of $2.2 \mathrm{~mm}$, and a glass top support. The parameters for the simulation were obtained by measuring the actual dimensions of patterned hydrogels. For the meshes, 4-node tetrahedral elements (SOLID72) were used. A grid size of $0.01 \mathrm{~mm}$ was used for the hydrogel and $0.02 \mathrm{~mm}$ for the other materials. A sensitivity analysis was performed to ensure that changes in the mesh size did not result in differences in deformations and stress and strain distributions. All parts were modeled as elastic flexible bodies with isotropic material properties. Young's modulus and Poisson's ratio of $5.0 \mathrm{kPa}$ and 0.49 $\mathrm{kPa}$, respectively, were used for the hydrogel, $1.84 \mathrm{MPa}$ and $0.49 \mathrm{MPa}$ were used for the PDMS structure, ${ }^{67}$ and $65 \mathrm{GPa}$ and $0.49 \mathrm{GPa}$ were used for the glass support. PDMS-hydrogel contact was modeled as frictionless, whereas hydrogel-top support contact was modeled as bonded. The base of the unit, as well as the top support, was constrained as fixed, and a linearly increasing pressure up to $14 \mathrm{kPa}$ was applied to the bottom surface of the PDMS membrane. The strains generated within the hydrogel sample were analyzed. To simulate the displacement of posts upon the deformation of the PDMS membrane, separate models were prepared consisting of only the PDMS structure with varying chamber diameters $(5,6,7$, and $8 \mathrm{~mm})$, membrane thicknesses (300, 450 , and $560 \mu \mathrm{m}$ ), and $\mathrm{N}_{2}$ pressures $(14,28$, and $42 \mathrm{kPa}$ ).

4.7. Biochemical Assay Analysis. Cell viability was evaluated over time (days 0, 3, 5, 7 and 21) using a LIVE/DEAD Viability/Cytotoxicity Kit (Invitrogen) according to the manufacturer's instructions $(N=5)$. The hydrogels were washed with DPBS twice and imaged using an inverted fluorescence microscope (Zeiss Axio Observer D1; Zeiss, Göttingen, Germany). The number of live and dead cells was counted for four samples of each group using ImageJ software (NIH). After 7 and 21 days culture, each hydrogel-cell construct was collected in CelLytic $\mathrm{M}$ solution (Sigma) and homogenized for $30 \mathrm{~s}$ using a TH homogenizer (Omni International, Marietta, GA, USA). Then, the homogenized solution was analyzed to measure DNA, ALP, and calcium content in each construct using Quant-iT PicoGreen dsDNA reagent kit (Invitrogen), ALP Assay kit (Sigma), and calcium assay kit (Pointe Scientific, Canton, MI, USA), respectively, according to the manufacturers' instructions. Fluorescence intensity of the dyeconjugated DNA solution was measured with a plate reader (Safire; Tecan, Austria), and the DNA content was calculated from a standard curve generated with Lambda DNA standard (Invitrogen). The absorbance of ALP and calcium assays was read at 405 and $570 \mathrm{~nm}$ on a plate reader and compared with standard curves prepared with 4nitrophenol standard solution (Sigma) and calcium standard solution 
(Sigma), respectively. ALP and calcium levels were measured and then the obtained data were normalized to the DNA content $(N=5)$.

4.8. Histological and Immunohistochemical Analysis. hMSCs encapsulated in GelMA hydrogels were collected $(N=4)$, washed with DPBS, and fixed with $4 \%$ paraformaldehyde (Sigma) solution for 20 min. To analyze cell spreading, samples were washed three times with DPBS and then incubated in $0.1 \%(\mathrm{w} / \mathrm{v}$ ) of Triton X-100 in DPBS for 20 $\mathrm{min}$ to make the cells permeable. Then, the samples were stained with Alexa Fluor 568 phalloidin for $30 \mathrm{~min}$ and followed by $30 \mathrm{~min}$ staining of 2-(4-amidinophenyl)-6-indolecarbamidine dihydrochloride (DAPI, Sigma). The stained cells were imaged using an inverted fluorescence microscope (Axio Observer D1, Zeiss) and a Leica SP5 confocal microscope (Leica Microsystems, Wetzlar, Germany). Cell spreading area and cell aspect ratio of phalloidin/DAPI-stained cells (number of cells $>30$ ) were analyzed using a custom algorithm in Image software. For the analysis of osteogenically differentiated cells, fixed samples $(N=$ 8) were embedded in an optimal cutting temperature compound (Fisher, Pittsburgh, PA), frozen, and cut into $20 \mu \mathrm{m}$ thick sections at $-20{ }^{\circ} \mathrm{C}$. Sectioned slides were washed three times with DPBS and stained with $2 \%$ alizarin red $\mathrm{S}$ solution ( $\mathrm{pH} 4.2$ ). After washing, the slides were dehydrated with a graded ethanol series and mounted with Permount mounting medium (Fisher). To evaluate OCN and OPN expression in the constructs, sections were stained with anti-OCN (ab93876, Abcam, Cambridge, UK) and anti-OPN (ab8448, Abcam) antibodies overnight at $4{ }^{\circ} \mathrm{C}$. Broad spectrum Histostain-Plus kit (Invitrogen) and aminoethyl carbazole (AEC; Invitrogen) were applied to visualize the staining. ${ }^{68}$ A negative control was prepared by applying secondary antibodies and AEC solution without any primary antibody treatment. A positive control was prepared by applying primary and secondary antibodies to bonelike engineered tissue sections from another study. ${ }^{69}$ Negative and positive controls were used to confirm no background staining and reactivity of antibodies. Slides were mounted with glycerol vinyl alcohol (Invitrogen) and imaged using an Olympus BX61VS microscope (Olympus, Tokyo, Japan).

4.9. Quantitative Real-Time Reverse Transcription Polymerase Chain Reaction (qRT-PCR). Cell-encapsulated samples were homogenized and lysed in TRI reagent (Sigma). Reverse transcription was performed using extracted RNA and iScript cDNA synthesis kit (Bio-Rad, Hercules, CA, USA) followed by qRT-PCR using primers human 3-phosphate dehydrogenase (GAPDH; forward primer 5'-GGG GCT GGC ATT GCC CTC AA-3'and reverse primer $5^{\prime}$-GGC TGG TGG TCC AGG GGT CT-3') and human Runx2 (forward primer 5'ACA GAA CCA CAA GTG CGG TGC AA-3' and reverse primer 5'TGG CTG GTA GTG ACC TGC GGA-3'). qRT-PCR was performed using Eppendorf Mastercycler (Eppendorf, Westbury, NY, USA), and GAPDH was served as an internal control. All data were analyzed using the $2^{-\Delta \Delta C_{\mathrm{t}}}$ method.

4.10. Statistical Analysis. All quantitative data are expressed as mean \pm standard deviation. Statistical analysis was performed with oneway analysis of variance with the Tukey significant difference post hoc test using Origin software (OriginLab Co., Northampton, MA, USA). A value of $p<0.05$ was considered statistically significant.

\section{ASSOCIATED CONTENT}

\section{S Supporting Information}

The Supporting Information is available free of charge on the ACS Publications website at DOI: 10.1021/acsami.7b17991.

Experimental and simulation data of the compressive strain with the pressure chamber diameter and the applied pressures as variables; combinatorial screening of $3 \mathrm{D}$ cellular behaviors of hMSCs by varying the hydrogel concentration and dynamic compression; and DNA content of hMSCs encapsulated in GelMA hydrogels after 7 and 21 days of osteogenic differentiation (PDF) Displacements of posts in the bioreactor during repeated cycles (AVI)

Cyclic deformation of GelMA hydrogel under dynamic compressive strains (AVI)

\section{AUTHOR INFORMATION}

\section{Corresponding Authors}

*E-mail: exa46@case.edu (E.A.).

*E-mail: khademh@ucla.edu (A.K.).

ORCID 웅

Eben Alsberg: 0000-0002-3487-4625

Ali Khademhosseini: 0000-0002-2692-1524

Author Contributions

$¥$ J.S. and J.-Y.S. contributed equally to this work.

Notes

The authors declare no competing financial interest.

\section{ACKNOWLEDGMENTS}

The authors gratefully acknowledge funding by the Defense Threat Reduction Agency under Space and Naval Warfare Systems Center Pacific contract no. N66001-13-C-2027. The authors also acknowledge funding from the National Institutes of Health (EB012597, AR057837, AR066193, DE021468, HL099073, and R56AI105024). Dr. Seo was partially supported by the Basic Science Research Program through the National Research Foundation of Korea funded by the Ministry of Education (2016R1A6A3A03006491) and KIST project (2E27930). Dr. Bal Ozturk was fully supported by post-doctoral research grant of The Scientific and Technological Research Council of Turkey (TUBITAK). J. L. acknowledges financial support from the Netherlands Organization for Scientific Research (NWO, Veni, \#14328), the European Research Council (ERC, Starting Grant, \#759425), and the Dutch Arthritis Foundation (\#17-1-405).

\section{REFERENCES}

(1) Discher, D. E.; Mooney, D. J.; Zandstra, P. W. Growth Factors, Matrices, and Forces Combine and Control Stem Cells. Science 2009, $324,1673-1677$.

(2) Lutolf, M. P.; Hubbell, J. A. Synthetic Biomaterials as Instructive Extracellular Microenvironments for Morphogenesis in Tissue Engineering. Nat. Biotechnol. 2005, 23, 47-55.

(3) Patwari, P.; Lee, R. T. Mechanical Control of Tissue Morphogenesis. Circ. Res. 2008, 103, 234-243.

(4) Morrison, S. J.; Spradling, A. C. Stem Cells and Niches: Mechanisms that Promote Stem Cell Maintenance throughout Life. Cell 2008, 132, 598-611.

(5) Sanz-Ezquerro, J. J.; Tickle, C. "Fingering" the Vertebrate Limb. Differentiation 2001, 69, 91-99.

(6) Allen, J. L.; Cooke, M. E.; Alliston, T. ECM Stiffness Primes the TGF $\beta$ Pathway to Promote Chondrocyte Differentiation. Mol. Biol. Cell 2012, 23, 3731-3742.

(7) Engler, A. J.; Sen, S.; Sweeney, H. L.; Discher, D. E. Matrix Elasticity Directs Stem Cell Lineage Specification. Cell 2006, 126, 677689.

(8) Fletcher, D. A.; Mullins, R. D. Cell Mechanics and the Cytoskeleton. Nature 2010, 463, 485-492.

(9) Cui, Y.; Hameed, F. M.; Yang, B.; Lee, K.; Pan, C. Q.; Park, S.; Sheetz, M. Cyclic Stretching of Soft Substrates Induces Spreading and Growth. Nat. Commun. 2015, 6, 6333.

(10) Vogel, V.; Sheetz, M. Local Force and Geometry Sensing Regulate Cell Functions. Nat. Rev. Mol. Cell Biol. 2006, 7, 265-275.

(11) Drury, J. L.; Mooney, D. J. Hydrogels for Tissue Engineering: Scaffold Design Variables and Applications. Biomaterials 2003, 24, 4337-4351.

(12) Bienaimé, C.; Barbotin, J.-N.; Nava-Saucedo, J.-E. How to Build an Adapted and Bioactive Cell Microenvironment? A Chemical Interaction Study of the Structure of Ca-alginate Matrices and Their Repercussion on Confined Cells. J. Biomed. Mater. Res., Part A 2003, 67, $376-388$. 
(13) Peppas, N. A.; Hilt, J. Z.; Khademhosseini, A.; Langer, R. Hydrogels in Biology and Medicine: from Molecular Principles to Bionanotechnology. Adv. Mater. 2006, 18, 1345-1360.

(14) Spitters, T. W. G. M.; Leijten, J. C. H.; Deus, F. D.; Costa, I. B. F.; van Apeldoorn, A. A.; van Blitterswijk, C. A.; Karperien, M. A Dual Flow Bioreactor with Controlled Mechanical Stimulation for Cartilage Tissue Engineering. Tissue Eng., Part C 2013, 19, 774-783.

(15) Oliveira, M. B.; Mano, J. F. High-Throughput Screening for Integrative Biomaterials Design: Exploring Advances and New Trends. Trends Biotechnol. 2014, 32, 627-636.

(16) Mei, Y.; Saha, K.; Bogatyrev, S. R.; Yang, J.; Hook, A. L.; Kalcioglu, Z. I.; Cho, S.-W.; Mitalipova, M.; Pyzocha, N.; Rojas, F. Combinatorial Development of Biomaterials for Clonal Growth of Human Pluripotent Stem Cells. Nat. Mater. 2010, 9, 768-778.

(17) Gobaa, S.; Hoehnel, S.; Roccio, M.; Negro, A.; Kobel, S.; Lutolf, M. P. Artificial Niche Microarrays for Probing Single Stem Cell Fate in high throughput. Nat. Methods 2011, 8, 949-955.

(18) Anderson, D. G.; Levenberg, S.; Langer, R. Nanoliter-Scale Synthesis of Arrayed Biomaterials and Application to Human Embryonic Stem Cells. Nat. Biotechnol. 2004, 22, 863-866.

(19) Anderson, D. G.; Putnam, D.; Lavik, E. B.; Mahmood, T. A.; Langer, R. Biomaterial Microarrays: Rapid, Microscale Screening of Polymer-Cell Interaction. Biomaterials 2005, 26, 4892-4897.

(20) Baharvand, H.; Hashemi, S. M.; Ashtiani, S. K.; Farrokhi, A. Differentiation of Human Embryonic Stem Cells into Hepatocytes in 2D and 3D Culture Systems in vitro. Int. J. Dev. Biol. 2004, 50, 645-652.

(21) Seo, J.; Lee, J. S.; Lee, K.; Kim, D.; Yang, K.; Shin, S.; Mahata, C.; Jung, H. B.; Lee, W.; Cho, S.-W.; Lee, T. Switchable Water-Adhesive, Superhydrophobic Palladium-Layered Silicon Nanowires Potentiate the Angiogenic Efficacy of Human Stem Cell Spheroids. Adv. Mater. 2014, 26, 7043-7050.

(22) Tian, X.-F.; Heng, B.-C.; Ge, Z.; Lu, K.; Rufaihah, A. J.; Fan, V. T.W.; Yeo, J.-F.; Cao, T. Comparison of Osteogenesis of Human Embryonic Stem Cells within 2D and 3D Culture Systems. Scand. J. Clin. Lab. Invest. 2008, 68, 58-67.

(23) Davisson, T.; Kunig, S.; Chen, A.; Sah, R.; Ratcliffe, A. Static and Dynamic Compression Modulate Matrix Metabolism in Tissue Engineered Cartilage. J. Orthop. Res. 2002, 20, 842-848.

(24) Rath, B.; Nam, J.; Knobloch, T. J.; Lannutti, J. J.; Agarwal, S. Compressive Forces Induce Osteogenic Gene Expression in Calvarial Osteoblasts. J. Biomech. 2008, 41, 1095-1103.

(25) Bian, L.; Fong, J. V.; Lima, E. G.; Stoker, A. M.; Ateshian, G. A.; Cook, J. L.; Hung, C. T. Dynamic Mechanical Loading Enhances Functional Properties of Tissue-Engineered Cartilage Using Mature Canine Chondrocytes. Tissue Eng., Part A 2010, 16, 1781-1790.

(26) Gaharwar, A. K.; Arpanaei, A.; Andresen, T. L.; Dolatshahi-Pirouz, A. 3D Biomaterial Microarrays for Regenerative Medicine: Current State-of-the-Art, Emerging Directions and Future Trends. Adv. Mater. 2016, 28, 771-781.

(27) Beachley, V. Z.; Wolf, M. T.; Sadtler, K.; Manda, S. S.; Jacobs, H.; Blatchley, M. R.; Bader, J. S.; Pandey, A.; Pardoll, D.; Elisseeff, J. H. Tissue Matrix Arrays for High-Throughput Screening and Systems Analysis of Cell Function. Nat. Methods 2015, 12, 1197-1204.

(28) Moraes, C.; Wang, G.; Sun, Y.; Simmons, C. A. A Microfabricated Platform for High-Throughput Unconfined Compression of Micropatterned Biomaterial Arrays. Biomaterials 2010, 31, 577-584.

(29) Liu, H.; Usprech, J.; Sun, Y.; Simmons, C. A. A Microfabricated Platform with Hydrogel Arrays for 3D Mechanical Stimulation of Cells. Acta Biomater. 2016, 34, 113-124.

(30) Li, Y.; Huang, G.; Gao, B.; Li, M.; Genin, G. M.; Lu, T. J.; Xu, F. Magnetically Actuated Cell-Laden Microscale Hydrogels for Probing Strain-Induced Cell Responses in Three Dimensions. NPG Asia Mater. 2016, 8, No. e238.

(31) Elsaadany, M.; Harris, M.; Yildirim-Ayan, E. Design and Validation of Equiaxial Mechanical Strain Platform, EQUicycler, for 3D Tissue Engineered Constructs. BioMed Res. Int. 2017, 2017, 3609703.

(32) Subramanian, G.; Elsaadany, M.; Bialorucki, C.; Yildirim-Ayan, E. Creating Homogenous Strain Distribution within 3D Cell-Encapsulated
Constructs Using a Simple and Cost-Effective Uniaxial Tensile Bioreactor: Design and Validation Study. Biotechnol. Bioeng. 2017, 114, 1878-1887.

(33) Hsieh, H.-Y.; Camci-Unal, G.; Huang, T.-W.; Liao, R.; Chen, T.-J.; Paul, A.; Tseng, F.-G.; Khademhosseini, A. Gradient Static-Strain Stimulation in a Microfluidic Chip for 3D Cellular Alignment. Lab Chip 2014, 14, 482-493.

(34) Lee, S. A.; Chung, S. E.; Park, W.; Lee, S. H.; Kwon, S. ThreeDimensional Fabrication of Heterogeneous Microstructures Using Soft Membrane Deformation and Optofluidic Maskless Lithography. Lab Chip 2009, 9, 1670-1675.

(35) Seo, J.; Lee, S.-K.; Lee, J.; Lee, J. S.; Kwon, H.; Cho, S.-W.; Ahn, J.H.; Lee, T. Path-Programmable Water Droplet Manipulations on an Adhesion Controlled Superhydrophobic Surface. Sci. Rep. 2015, 5, 12326.

(36) Dolatshahi-Pirouz, A.; Nikkhah, M.; Gaharwar, A. K.; Hashmi, B.; Guermani, E.; Aliabadi, H.; Camci-Unal, G.; Ferrante, T.; Foss, M.; Ingber, D. E.; Khademhosseini, A. A Combinatorial Cell-Laden Gel Microarray for Inducing Osteogenic Differentiation of Human Mesenchymal Stem Sells. Sci. Rep. 2014, 4, 3896.

(37) Scott, A.; Khan, K.; Heer, J.; Cook, J.; Lian, O.; Duronio, V. High Strain Mechanical Loading Rapidly Induces Tendon apoptosis: an ex vivo Rat Tibialis Anterior Model. Br. J. Sports Med. 2005, 39, No. e25.

(38) Appleby-Thomas, G. J.; Hazell, P. J.; Sheldon, R. P.; Stennett, C.; Hameed, A.; Wilgeroth, J. M. The High Strain-Rate Behaviour of Selected Tissue Analogues. J. Mech. Behav. Biomed. Mater. 2014, 33, 124-135.

(39) Pioletti, D. P.; Rakotomanana, L. R.; Leyvraz, P.-F. Strain Rate Effect on the Mechanical Behavior of the Anterior Cruciate LigamentBone Complex. Med. Eng. Phys. 1999, 21, 95-100.

(40) Prevost, T. P.; Balakrishnan, A.; Suresh, S.; Socrate, S. Biomechanics of Brain Tissue. Acta Biomater. 2011, 7, 83-95.

(41) Krishnamurthy, G.; Itoh, A.; Bothe, W.; Swanson, J. C.; Kuhl, E.; Karlsson, M.; Miller, D. C.; Ingels, N. B. Stress-Strain Behavior of Mitral Valve Leaflets in the Beating Ovine Heart. J. Biomech. 2009, 42, 1909-1916.

(42) van Sligtenhorst, C.; Cronin, D. S.; Brodland, G. W. High Strain Rate Compressive Properties of Bovine Muscle Tissue Determined Using a Split Hopkinson Bar Apparatus. J. Biomech. 2006, 39, 18521858.

(43) del Campo, A.; Greiner, C. SU-8: a Photoresist for High-AspectRatio and 3D Submicron Lithography. J. Micromech. Microeng. 2007, 17, R81.

(44) Larrañeta, E.; Lutton, R. E. M.; Woolfson, A. D.; Donnelly, R. F. Microneedle Arrays as Transdermal and Intradermal Drug Delivery Systems: Materials Science, Manufacture and Commercial Development. Mater. Sci. Eng. R Rep. 2016, 104, 1-32.

(45) Varga, B.; Fazakas, C.; Wilhelm, I.; Krizbai, I. A.; Szegletes, Z.; Váró, G.; Végh, A. G. Elasto-Mechanical Properties of Living Cells. Biochem. Biophys. Rep. 2016, 7, 303-308.

(46) Hwang, C. M.; Sant, S.; Masaeli, M.; Kachouie, N. N.; Zamanian, B.; Lee, S.-H.; Khademhosseini, A. Fabrication of Three-Dimensional Porous Cell-Laden Hydrogel for Tissue Engineering. Biofabrication 2010, 2, 035003.

(47) Hutson, C. B.; Nichol, J. W.; Aubin, H.; Bae, H.; Yamanlar, S.; AlHaque, S.; Koshy, S. T.; Khademhosseini, A. Synthesis and Characterization of Tunable Poly (ethylene glycol): Gelatin Methacrylate Composite Hydrogels. Tissue Eng., Part A 2011, 17, 1713-1723.

(48) McBeath, R.; Pirone, D. M.; Nelson, C. M.; Bhadriraju, K.; Chen, C. S. Cell Shape, Cytoskeletal Tension, and RhoA Regulate Stem Cell Lineage Commitment. Dev. Cell 2004, 6, 483-495.

(49) Dupont, S.; Morsut, L.; Aragona, M.; Enzo, E.; Giulitti, S.; Cordenonsi, M.; Zanconato, F.; Le Digabel, J.; Forcato, M.; Bicciato, S.; Elvassore, N.; Piccolo, S. Role of YAP/TAZ in Mechanotransduction. Nature 2011, 474, 179-183.

(50) Cukierman, E.; Pankov, R.; Yamada, K. M. Cell Interactions with Three-Dimensional Matrices. Curr. Opin. Cell Biol. 2002, 14, 633-639. 
(51) Baker, B. M.; Chen, C. S. Deconstructing the Third Dimension: How 3D Culture Microenvironments Alter Cellular Cues. J. Cell Sci. 2012, 125, 3015-3024.

(52) Sikavitsas, V. I.; Temenoff, J. S.; Mikos, A. G. Biomaterials and bone mechanotransduction. Biomaterials 2001, 22, 2581-2593.

(53) Gurkan, U. A.; Akkus, O. The Mechanical Environment of Bone Marrow: a Review. Ann. Biomed. Eng. 2008, 36, 1978-1991.

(54) Shachar, M.; Benishti, N.; Cohen, S. Effects of Mechanical Stimulation Induced by Compression and Medium Perfusion on Cardiac Tissue Engineering. Biotechnol. Prog. 2012, 28, 1551-1559.

(55) Powell, C. A.; Smiley, B. L.; Mills, J.; Vandenburgh, H. H. Mechanical Stimulation Improves Tissue-Engineered Human Skeletal Muscle. Am. J. Physiol. 2002, 283, C1557-C1565.

(56) Elder, B. D.; Athanasiou, K. A. Hydrostatic Pressure in Articular Cartilage Tissue Engineering: from Chondrocytes to Tissue Regeneration. Tissue Eng., Part B 2009, 15, 43-53.

(57) Wang, J.; Wang, C. D.; Zhang, N.; Tong, W. X.; Zhang, Y. F.; Shan, S. Z.; Zhang, X. L.; Li, Q. F. Mechanical Stimulation Orchestrates the Osteogenic Differentiation of Human Bone Marrow Stromal Cells by Regulating HDAC1. Cell Death Dis. 2016, 7, No. e2221.

(58) Granéli, C.; Thorfve, A.; Ruetschi, U.; Brisby, H.; Thomsen, P.; Lindahl, A.; Karlsson, C. Novel Markers of Osteogenic and Adipogenic Differentiation of Human Bone Marrow Stromal Cells Identified Using a Quantitative Proteomics Approach. Stem Cell Res. 2014, 12, 153-165.

(59) Zhang, P.; Wu, Y.; Jiang, Z.; Jiang, L.; Fang, B. Osteogenic Response of Mesenchymal Stem Cells to Continuous Mechanical Strain is Dependent on ERK1/2-Runx2 Signaling. Int. J. Mol. Med. 2012, 29, 1083-1089.

(60) Kanno, T.; Takahashi, T.; Tsujisawa, T.; Ariyoshi, W.; Nishihara, T. Mechanical Stress-Mediated Runx2 Activation is Dependent on Ras/ ERK1/2 MAPK Signaling in Osteoblasts. J. Cell. Biochem. 2007, 101, $1266-1277$.

(61) Kermani, S.; Wahab, R. M. A.; Abidin, I. Z. Z.; Ariffin, Z. Z.; Senafi, S.; Ariffin, S. H. Z. Differentiation Capacity of Mouse Dental Pulp Stem Cells into Osteoblasts and Osteoclasts. Cell J. 2014, 16, 31.

(62) Miura, M.; Chen, X.-D.; Allen, M. R.; Bi, Y.; Gronthos, S.; Seo, B.M.; Lakhani, S.; Flavell, R. A.; Feng, X.-H.; Robey, P. G.; Young, M.; Shi, S. A Crucial Role of Caspase-3 in Osteogenic Differentiation of Bone Marrow Stromal Stem Cells. J. Clin. Invest. 2004, 114, 1704-1713.

(63) Kuo, Z.-K.; Lai, P.-L.; Toh, E. K.-W.; Weng, C.-H.; Tseng, H.-W.; Chang, P.-Z.; Chen, C.-C.; Cheng, C.-M. Osteogenic Differentiation of Preosteoblasts on a Hemostatic Gelatin Sponge. Sci. Rep. 2016, 6, 32884 .

(64) Nichol, J. W.; Koshy, S. T.; Bae, H.; Hwang, C. M.; Yamanlar, S.; Khademhosseini, A. Cell-Laden Microengineered Gelatin Methacrylate Hydrogels. Biomaterials 2010, 31, 5536-5544.

(65) Chen, E. J.; Novakofski, J.; Jenkins, W. K.; O’Brien, W. D. Young's Modulus Measurements of Soft Tissues with Application to Elasticity Imaging. IEEE Trans. Son. Ultrason. 1996, 43, 191-194.

(66) Haynesworth, S. E.; Goshima, J.; Goldberg, V. M.; Caplan, A. I. Characterization of Cells with Osteogenic Potential from Human Marrow. Bone 1992, 13, 81-88.

(67) Sinha, R.; Le Gac, S.; Verdonschot, N.; van den Berg, A.; Koopman, B.; Rouwkema, J. Medium Throughput Device to Study the Effects of Combinations of Surface Strains and Fluid-Flow Shear Stresses on Cells. Lab Chip 2015, 15, 429-439.

(68) Estes, B. T.; Diekman, B. O.; Gimble, J. M.; Guilak, F. Isolation of Adipose-Derived Stem Cells and Their Induction to a Chondrogenic Phenotype. Nat. Protoc. 2010, 5, 1294-1311.

(69) Dang, P. N.; Dwivedi, N.; Phillips, L. M.; Yu, X.; Herberg, S.; Bowerman, C.; Solorio, L. D.; Murphy, W. L.; Alsberg, E. Controlled Dual Growth Factor Delivery from Microparticles Incorporated within Human Bone Marrow-Derived Mesenchymal Stem Cell Aggregates for Enhanced Bone Tissue Engineering via Endochondral Ossification. Stem Cells Transl. Med. 2016, 5, 206-217. 